\title{
Comparing Self-Selected Speed Walking of the Elderly With Self-Selected Slow, Moderate, and Fast Speed Walking of Young Adults
}

\author{
Woo Sub Kim, MD, Eun Young Kim, MD
}

Department of Rehabilitation Medicine, Veterans Health Service Medical Center, Seoul, Korea

\begin{abstract}
Objective To find the characteristics of elderly gait, we compared the elderly walking at a moderate speed with the young adult walking at a slow, moderate, and fast speed.

Methods 3D gait analysis was performed on 15 elderly and 15 young adults. Temporo-spatial, kinematic, and kinetic parameters were obtained. Self-selected moderate speed of the elderly walking was compared with selfselected varying speed of the young adults walking.

Results The elderly walked at slower speeds and had shorter step length, but showed similar cadences compared to the young adults. These results remained identical even after the normalization with height. The kinematic and the kinetic graph patterns did not show specific differences between the elderly and the young subjects. Ankle plantarflexion (APF) motion was prominently decreased in the elderly subjects. Hip flexion (HF) motion remained within similar range for the young adults. HF moment and power were similar with the young adults', but APF power and hip extension power were decreased in the elderly subjects'.

Conclusion A decreased APF motion and power were thought to be specific findings in the elderly walking. The preservation of HF motion and power could be considered a compensation mechanism or a modified neuromuscular pattern in the elderly. The characteristics of the elderly walking should be taken into account when planning rehabilitation strategies of elderly gait training and for future studies on the elderly population.
\end{abstract}

Keywords Aging, Gait

Received March 8, 2013; Accepted August 27, 2013

Corresponding author: Eun Young Kim

Department of Rehabilitation Medicine, Veterans Health Service Medical Center, 53 Jinhwangdo-ro 61-gil, Gangdong-gu, Seoul 134-791, Korea

Tel: +82-2-2225-1535, Fax: +82-2-2225-1535, E-mail: knox79@hanmail. net

(ㄷ) This is an open-access article distributed under the terms of the Creative Commons Attribution Non-Commercial License (http://creativecommons. org/licenses/by-nc/3.0) which permits unrestricted noncommercial use, distribution, and reproduction in any medium, provided the original work is properly cited.

Copyright $\odot 2014$ by Korean Academy of Rehabilitation Medicine

\section{INTRODUCTION}

Aging inevitably accompanies changes in the musculoskeletal and the neural system [1]. Muscle, ligament, tendon, cartilage, peripheral and the central nerve system go through anatomical and physiological changes with aging. Weaknesses in muscle are caused by the loss of muscle fibers, a decrease in the aerobic capacity, and diminished motor neurons [2]. Increased cross links in the soft tissue around the joint decreases the flexibility of the soft tissue and induce joint stiffness [3]. Osteophytes and 
joint incongruities provoked by the excessive joint wearing at articular surfaces could be a source of limitation in the joint motion. The deterioration of the balance and the coordination are associated with the loss of neurons, an increased reaction time, a reduced neurotransmitter production, and a decrease in a number of sensory functions including vestibular, visual, and somatosensory.

The neuromuscular changes associated with aging induce the limitation of functional activities, such as walking and posture. The elderly experience their posture becoming more bent; the knee and the hip get more flexed and the neck and the shoulder become more tilted and narrowed. The elderly walk at a slower speed and have shorter step length than the young adults. The changes in the posture and walking are related to the musculoskeletal pain, a decreased cardiopulmonary function, and an increased risk of falling.

Many researchers have studied the elderly walking in order to identify characteristic patterns, limiting factors, the adaptation and compensation mechanisms. A decrease in the ankle plantar flexor (APF) power, a limitation in the hip extension range and the compensation by hip muscles have been found to be the distinguishing features of the elderly walking from the walking of young adults [4-8]. Prince et al. [1] reported that the characteristic findings of the elderly walking were a decreased stride length, a significantly lower APF power in the terminal stance, and the reduction in the knee absorption power. Kerrigan et al. [5] reported the hip flexor contracture and the APF concentric weakness as the limiting factors in the elderly. DeVita and Hortobagyi [6] reported that the elderly have more power at the hip extensor and less power at the knee and the ankle based on the speed matched experiments between the elderly and the young adults.

The slowing of a preferred walking is a common finding in the studies on the elderly walking. The step length and the cadence showed a positive correlation with the walking speed in the studies of the intra-personal speed change [9]. Although a decreased walking speed and step length have been observed consistently, the cadence did not decrease in some comparison studies between the elderly and the young age groups. Although the temporospatial parameters are not only easy to obtain but also representative of the walking function, the changes in the temporo-spatial parameters are associated with the changes in the kinematic or the kinetic parameters $[10,11]$ and can be a confounding factor for interpreting changes in the other parameters. It has not been clear whether the changes in the gait parameters, such as the joint angle, the joint moment and the joint power, and the power of the elderly, are due to aging or the slowing of walking speed. The goal of this study was to compare healthy elderly men walking at a self-selected moderate speed with young men walking at self-selected slow, moderate, and fast speeds, and to determine the specific findings of the elderly walking.

\section{MATERIALS AND METHODS}

The gait data for the 15 healthy young adults and the 15 elderly adults were analyzed. All subjects were male and had no functional limitations in daily activities. The young subjects were either hospital workers or students and they volunteered this study. The elderly subjects were recruited from the Veterans Health Service Medical Center rehabilitation department outpatient clinic and had no medical history limiting a safe level walking. The physiatrists excluded those subjects with neurologic or orthopedic diagnosis that could change the gait patterns and limit level walking. The elderly subjects with a medical history of brain or spinal cord abnormalities, an operation for lower extremity joint, an apparent lower extremity deformity, a peripheral neuropathy, or a radiculopathy causing lower extremity motor weakness, were excluded. This study was approved by Institutional Review Board of Veterans Health Service Medical Center.

The subjects were first asked to stand for static trial and then walked barefoot across the $8 \mathrm{~m}$ walkway for dynamic trials. Infrared reflective markers were attached to the subjects' lower extremity with the Helen Hayes marker system. An optoelectronic motion analysis system with five video cameras was used to measure the $3 \mathrm{D}$ trajectories of the reflective markers. The ground reaction forces were measured synchronously with the kinematic data using two force platforms (OR6-5; Advanced Mechanical Technology Inc., Watertown, MA, USA) imbedded in the walkway with $60 \mathrm{~Hz}$ frequency.

Visual3D (C-motion Inc., Rockville, MD, USA) software was used to calculate the temporo-spatial, kinematic, and kinetic parameters with the C3D formatted motion files constructed by Vicon Workstation 512 system (Oxford Metrics Ltd., Oxford, UK). The gait cycle events and 
phases were determined by the ground reaction vector and the heel marker trajectories. The coordinate data of the reflective marker trajectory and the force plate data were low pass filtered with $6 \mathrm{~Hz}$ cutoff frequency. The

Table 1. Age, height, and weight of the subjects

\begin{tabular}{lcc}
\hline & Elderly & Young \\
\hline Age & $63.60 \pm 2.47^{*}$ & $27.06 \pm 2.89$ \\
Height $(\mathrm{cm})$ & $166.86 \pm 4.37^{*}$ & $174.86 \pm 4.64$ \\
\hline Weight $(\mathrm{kg})$ & $61.73 \pm 7.25^{*}$ & $71.33 \pm 9.28$ \\
\hline
\end{tabular}

Values are mean \pm one standard deviation.

${ }^{*} \mathrm{p}<0.05$, statistical significance. kinematic and the kinetic parameters were calculated by the inverse-dynamics method. The kinematic data were normalized with respect to the static trial posture. The kinetic data, internal moment and the joint power, were normalized by the subjects' weight.

The elderly subjects were asked to walk at their selfselected moderate speed. The young subjects walked at a self-selected moderate, fast, and slow speed. At least three trials were performed at each speed and more than two trials with a clean force plate contact were analyzed to calculate the kinematic and the kinetic data for each speed. The ensembles for the kinematic and the kinetic data were graphed to represent each subject's gait pat-
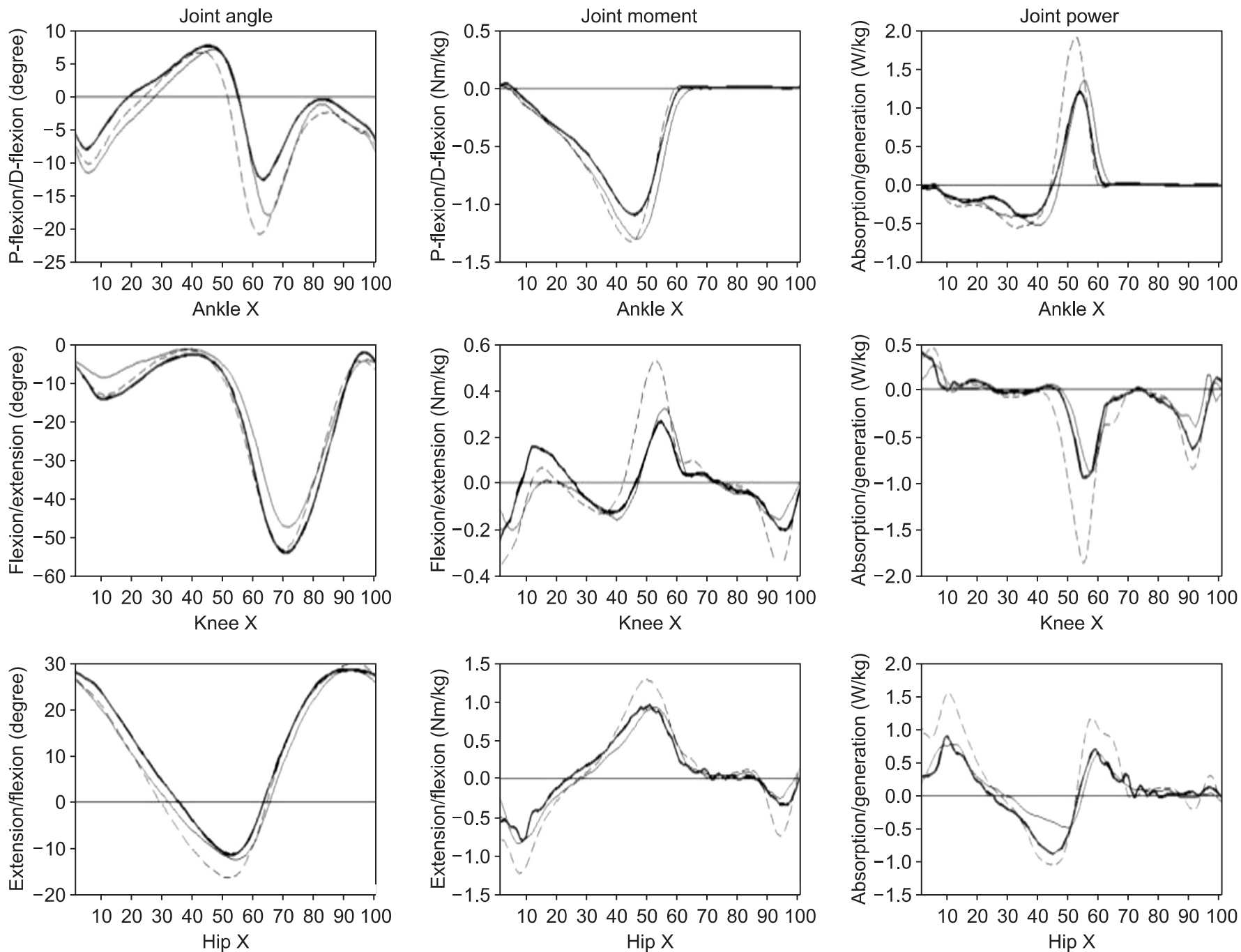

Fig. 1. The ensembles of the kinematic and the kinetic parameters are overlapped. The bold line indicates the elderly walking at a preferred moderate speed. The dotted line indicates the young adult walking at a preferred moderate speed. The plain line indicates the young adult walking at a preferred slow speed. The fast speed of the young adult walking is not overlapped for a clear comparison. 
tern.

The kinematic and the kinetic parameters of the elderly (ED), the young slow (YS), the young moderate (YM), and the young fast (YF) were statistically analyzed using the ANOVA test with Bonferroni adjustment to compare the elderly walking with the young walking.

\section{RESULTS}

The elderly subjects' ages ranged from 60 to 69 years (the average, $63.60 \pm 2.47$ years). The young adult subjects' age were from 24 to 34 years (average, 27.06 \pm 2.89 years). The elderly subjects were shorter in terms of height (166.86 \pm 4.37 vs. $174.86 \pm 4.64 ; \mathrm{p}<0.01)$ and had a lower weight ( $61.73 \pm 7.25$ vs. $71.33 \pm 9.28 ; \mathrm{p}<0.01)$ with statistical significances (Table 1). The patterns of joint angles, joint moments, and joint powers are reported by the ensemble mean graphs in Fig. 1.

\section{Temporo-spatial parameters}

The speed of ED was significantly slower than the speed of YM (1.00 \pm 0.12 vs. $1.27 \pm 0.10 ; \mathrm{p}<0.01)$ and significantly faster than the speed of YS $(1.00 \pm 0.12 \mathrm{vs}$. $0.85 \pm 0.15 ; p=0.0152)$. The step length of ED was similar to the step length of YS $(0.57 \pm 0.04$ vs. $0.57 \pm 0.05)$ and significantly shorter than the step length of YM $(0.57 \pm 0.04$ vs. $0.67 \pm 0.03 ; \mathrm{p}<0.01)$. The cadence of ED was significantly higher than that of YS (106.5 \pm 7.9 vs. $87.2 \pm 11.2$; $\mathrm{p}<0.01)$ and similar to the cadence of YM $(106.5 \pm 7.9$ vs. $112.4 \pm 5.2)$. The stance ratio during the gait cycle was similar between ED and YS $(0.58 \pm 0.00$ vs. $0.57 \pm 0.01)$. The normalization of the walking speed and the step length by each subject's height did not produce any changes in the statistical results (Table 2).

\section{Joint angles}

The joint angle results are listed in Table 3. The APF of ED showed a significantly reduced maximum angle compared with even that of YS $(12.7 \pm 7.9$ vs. $19.5 \pm 6.3$; $\mathrm{p}=0.0169$ ). The ADF of ED showed an increased maximum angle but the differences were not statistically significant. The hip flexion maximum angles were similar across the conditions but the hip extension maximum angle of ED was similar to that of YS $(11.0 \pm 4.0$ vs. $12.7 \pm 3.3)$ and smaller than that of YM with statistical significance (11.0 \pm 4.0 vs. $16.1 \pm 3.3 ; p=0.002$ ). Our observation of the knee flexion at the initial contact and the loading phase showed a similarity between ED and YM (Table 3 ).

\section{Joint moments}

The joint moment results are listed in Table 4. The APF moment of ED was slightly smaller than that of YS but differences across the conditions did not show any statistical significance. The hip extension moment of ED was smaller than that of YM $(0.89 \pm 0.37$ vs. $1.28 \pm 0.25 ; \mathrm{p}=$ $0.008)$ and similar to YS $(0.89 \pm 0.37$ vs. $0.77 \pm 0.47)$. The hip flexion moment of ED had no statistical differences with those of YS and YM (Table 4).

\section{Joint powers}

The APF power burst (A2) of ED was smaller than that of YM ( $1.10 \pm 0.12$ vs. $1.30 \pm 0.29 ; \mathrm{p}=0.017)$ and similar to that of YS. The ankle energy absorptions (A1) at stance phase were similar between ED and YS with no statistically sig-

Table 2. Temporo-spatial results of the elderly and the young subjects with speed changes

\begin{tabular}{lcccc} 
& Elderly & \multicolumn{3}{c}{ Young } \\
\cline { 2 - 4 } & & Slow & Moderate & Fast \\
\hline Speed (m/s) & $1.01 \pm 0.12$ & $0.83 \pm 0.14^{*}$ & $1.27 \pm 0.10^{*}$ & $1.58 \pm 0.15^{*}$ \\
Cadence & $107.38 \pm 7.64$ & $85.93 \pm 10.24^{*}$ & $112.66 \pm 5.19$ & $129.26 \pm 6.15^{*}$ \\
\hline Step length (m) & $0.57 \pm 0.04$ & $0.57 \pm 0.05$ & $0.67 \pm 0.04^{*}$ & $0.73 \pm 0.05^{*}$ \\
Stance ratio (\%) & $0.57 \pm 0.00$ & $0.57 \pm 0.01$ & $0.55 \pm 0.01^{*}$ & $0.54 \pm 0.01^{*}$ \\
Speed_NL (m/s) & $0.60 \pm 0.07$ & $0.47 \pm 0.07^{*}$ & $0.72 \pm 0.05^{*}$ & $0.89 \pm 0.08^{*}$ \\
Step length_NL (m) & $0.34 \pm 0.02$ & $0.32 \pm 0.02$ & $0.38 \pm 0.02^{*}$ & $0.41 \pm 0.03^{*}$ \\
\hline
\end{tabular}

Values are presented as mean \pm one standard deviation.

_NL means normalized results by the subject's height.

* indicates groups that showed a significant statistical difference with the elderly group after ANOVA and Bonferroni adjustment for multiple comparisons. 
Table 3. Joint angles of the elderly and the young subjects with speed changes (unit, degree)

\begin{tabular}{lrccc}
\hline & Elderly & \multicolumn{3}{c}{ Young } \\
\cline { 2 - 4 } & & Slow & Moderate & Fast \\
\hline Ankle DF & $8.0 \pm 2.8$ & $7.2 \pm 4.0$ & $6.8 \pm 4.1$ & $6.4 \pm 3.8$ \\
\hline Ankle PF & $12.7 \pm 7.9$ & $19.5 \pm 6.3^{*}$ & $22.3 \pm 5.0^{*}$ & $23.2 \pm 5.2^{*}$ \\
\hline Ankle range & $20.7 \pm 6.8$ & $26.7 \pm 3.5^{*}$ & $29.1 \pm 2.4^{*}$ & $29.6 \pm 4.2^{*}$ \\
\hline Hip flexion & $28.1 \pm 4.3$ & $26.2 \pm 3.9$ & $26.8 \pm 4.6$ & $29.0 \pm 5.0$ \\
\hline Hip extension & $11.0 \pm 4.0$ & $12.7 \pm 3.3$ & $16.1 \pm 3.3^{*}$ & $17.4 \pm 4.3^{*}$ \\
\hline Hip range & $39.2 \pm 4.6$ & $39.0 \pm 2.2$ & $42.9 \pm 3.1^{*}$ & $46.4 \pm 5.1^{*}$ \\
\hline Knee at IC & $5.7 \pm 5.7$ & $4.5 \pm 5.2$ & $5.7 \pm 4.4$ & $8.3 \pm 4.4$ \\
\hline Knee flexion & $14.3 \pm 5.0$ & $8.6 \pm 6.8^{*}$ & $13.8 \pm 7.6$ & $17.6 \pm 6.2$ \\
\hline Knee range & $8.6 \pm 4.5$ & $4.0 \pm 2.7^{*}$ & $7.6 \pm 4.1$ & $9.2 \pm 4.1$ \\
\hline
\end{tabular}

Values are presented as mean \pm one standard deviation.

Ankle DF is the maximum angle at stance phase. Ankle PF is the maximum angle at push off. Ankle range is the difference between the Ankle DF and the PF. Hip flexion is the maximum angle at IC. Hip extension is the maximum angle at terminal stance. Hip range is difference between the hip flexion and the extension. The knee flexion is the maximum angle at loading phase. The knee range is the difference between the knee flexion and the knee IC.

DF, dorsiflexion; PF, plantarflexion; IC, initial contact.

* indicates groups that showed a significant statistical difference from the elderly group after ANOVA and Bonferroni adjustment for multiple comparisons.

Table 4. Joint moments of the elderly and the young subjects with speed changes (unit, $\mathrm{Nm} / \mathrm{kg}$ )

\begin{tabular}{lcccc} 
& \multirow{2}{*}{ Elderly } & \multicolumn{3}{c}{ Young } \\
\cline { 3 - 4 } & & Slow & Moderate & Fast \\
\hline Ankle PF & $1.10 \pm 0.12$ & $1.20 \pm 0.23$ & $1.30 \pm 0.29$ & $1.33 \pm 0.27$ \\
Hip flexion & $1.01 \pm 0.31$ & $1.15 \pm 0.35$ & $1.33 \pm 0.42$ & $1.67 \pm 0.43^{*}$ \\
Hip extension & $0.89 \pm 0.37$ & $0.77 \pm 0.47$ & $1.28 \pm 0.25^{*}$ & $1.71 \pm 0.31^{*}$ \\
Knee extension & $0.18 \pm 0.10$ & $0.11 \pm 0.22$ & $0.15 \pm 0.29$ & $0.31 \pm 0.33$ \\
\hline
\end{tabular}

Values are presented as mean \pm one standard deviation.

Ankle moment is measured at the maximum value at push off. Hip extension moment is measured at the maximum at the early stance. Hip flexion moment is measured at the maximum around push off.

$\mathrm{PF}$, plantarflexion.

* indicates groups that showed a significant statistical difference from the elderly group after ANOVA and Bonferroni adjustment for multiple comparisons.

nificant differences across the conditions. The hip power exerted by the hip extensor at the loading phase (H1) was reduced in ED compared to that of YM (0.96 \pm 0.58 vs. $1.71 \pm 0.54 ; \mathrm{p}=0.044)$ and similar to that of YS $(0.96 \pm 0.58$ vs. $0.90 \pm 0.34$ ). The hip flexor power burst (H3) of ED was smaller than that of YM $(1.01 \pm 0.27$ vs. $1.25 \pm 0.39)$ and larger than that of YS $(1.01 \pm 0.27$ vs. $0.68 \pm 0.34)$ but there was no statistical significance across the conditions (Table 5).

\section{DISCUSSION}

The elderly subjects showed a short step length, a similar cadence and a slow walking speed compared to the young subjects at a preferred moderate walking speed. In order to rule out the effect of height, we normalized our basic temporo-spatial data with height, and we found no changes in the statistical results.

The young adult subjects showed diminished kinematic and kinetic ankle parameters at a decreasing walk- 
Table 5. Joint powers of the elderly and the young subjects with speed changes (unit, $\mathrm{W} / \mathrm{kg}$ )

\begin{tabular}{ccccc}
\hline & Elderly & \multicolumn{3}{c}{ Young } \\
\cline { 3 - 5 } & & Slow & Moderate & Fast \\
\hline A1 & $-0.59 \pm 0.18$ & $-0.59 \pm 0.40$ & $-0.72 \pm 0.49$ & $-0.80 \pm 0.42$ \\
A2 & $1.33 \pm 0.38$ & $1.43 \pm 0.45$ & $2.25 \pm 0.75^{*}$ & $3.28 \pm 1.46^{*}$ \\
H1 & $0.96 \pm 0.58$ & $0.90 \pm 0.34$ & $1.71 \pm 0.54^{*}$ & $2.35 \pm 1.37^{*}$ \\
H2 & $-0.95 \pm 0.39$ & $-0.79 \pm 0.38$ & $-1.19 \pm 0.66$ & $-1.77 \pm 0.86^{*}$ \\
H3 & $1.01 \pm 0.27$ & $0.68 \pm 0.34$ & $1.25 \pm 0.39$ & $2.55 \pm 1.45^{*}$ \\
\hline
\end{tabular}

Values are mean \pm one standard deviation.

Power was resolved to coordinate the axis of the lower limb segments with three components. We reported the mediolateral axis component of resolved power. Al means the ankle power absorption before the ankle power burst. A2 means the ankle power generation at push off. $\mathrm{Hl}$ means the power generation of the hip extensor at the early stance. $\mathrm{H} 2$ means the power absorption of the hip flexor during the stance phase. H3 means the power generation of the hip flexor for the progression at the toe off.

* indicates groups that showed a significant statistical difference from the elderly group after ANOVA and Bonferroni adjustment for multiple comparisons.

ing speed. The elderly subjects with a moderate speed showed a decreased APF power as small as that of the young adult subjects at a slow speed. The ankle range of motion in the elderly was smaller than that of the young adults at a slow speed.

The young adult subjects showed diminished kinematic and kinetic hip parameters at a decreasing walking speed. The elderly subjects showed the hip total range of motion, the extension range of motion, and the extensor kinetics, decreased at the level of the young adults at a slow speed. However, the flexion range of motion and the flexor power did not show statistically significant differences with the young adult at a moderate speed.

A research on the elderly should consider changes in the gait parameters provoked by the confounding factors, such as aging, the walking speed, the cultural background, the laboratory settings, the gait analysis marker placement, and the calculation algorithms. The gait parameters observed during the gait cycle change in accordance with the walking speed. Lelas et al. [10] reported that the kinetic and the kinematic parameters showed a linear or a non-linear relationship with the walking speed. These relationships make it difficult to interpret the 3D gait analysis reports and many researchers have tried to control the walking speed in their experiments. There have been several methods, such as self-selected speeds, pre-determined speeds, and statistical control for controlling the walking speeds. Yet, each method has advantages and disadvantages; the pre-determined speed control trials have the disadvantage of capturing the un- natural and the poor repeatable mechanisms [12]. We thought that the subjects would perform different tasks during the pre-determined speed control; for example, a $1 \mathrm{~m} / \mathrm{s}$ velocity walking would be a slow walking for some individuals while it would be a preferred speed for other individuals. Astephen Wilson [12] suggested that a varying speed of self-selected walking in the gait experiments could provide an insight into the biomechanical aspects of walking in addition to the effect of the walking speed. We varied the walking speed of young adults at a fast, moderate, and slow speed and compared the moderate speed elderly walking to young adults', especially between the elderly and the slow walking of young adults; we expected to have findings that could not be explained merely by the walking speed changes.

Given the fact that the elderly subjects walked faster than the slow walking young adult subjects as well as the elderly subjects' preserved APF moment, we may conclude that the elderly have changes in their APF muscle function that are related to the kinematic function. The slowing of the walking speed cannot fully explain the decrease in the APF power and kinematic change in the elderly subjects. Prince et al. [1] and Winter et al. [4] reported that the elderly walked with a decreased APF power burst and interpreted the APF function as a forward progression of the body and suggested that the destabilization by a forward acceleration was responsible for the reduction of the ankle power. They interpreted these as an attempt to maintain the stability. Kerrigan et al. [5] indicated that the decrease in the APF power did 
not stem from the limitation in the range of the joint motion. They suggested dynamic stiffness or co-contraction of the ankle dorsiflexor as mechanisms of the APF power weakness due to the similarity in the APF moment with the young adults and the preservation of the eccentric contractility. They also suggested other possibilities for the APF power weakness, which increase the base of support to improve the stability. Our elderly subjects showed an ankle function deterioration, especially related to the kinematic properties that have a limitation in generating a rapid torque.

Considering the decreased walking speed, we believed that the preservation of the hip flexion range and the power would be specific findings that could not be explained by the decreasing walking speed in our elderly subjects. Judge et al. [13] observed a disappearance in the APF power weakness and an increased hip flexor power after adjusting the step length differences. They suggested that the increase of the hip flexor power would be a compensation mechanism in the elderly. DeVita and Hortobagyi [6] performed a speed matching study with the elderly and young adults and reported an increased hip extensor power in the elderly; they introduced the concept of 'distal to proximal shift of lower extremity function' in the elderly. McGibbon [8] supported the concept of a modified neuromuscular patterning in the elderly and suggested two possible adaptation mechanisms, one for the trunk stabilization by the hip extensor, another for the limb progression by the hip flexion. They compared the healthy elderly with the impaired elderly and reported a hip energy absorption as a compensation mechanism used by the impaired elderly. Kerrigan et al. [5] compared a moderate speed with a fast speed and presented that limiting factors that remained the same after a speed increase included the hip extension range in the elderly. They speculated that the hip flexor contracture was the cause of the limitation at the hip extensor.

We could not find out the limiting factors that are responsible for changing gait pattern in the Korean elderly. Based on our study, we thought that a decreased APF power and a maintained hip flexor power correspond with the concept of 'distal to proximal shift' proposed by Devita and Hortobagyi [6]. Decreased APF powers in the Korean elderly were related to the kinematic dysfunction associated with a difficulty of rapid torque. The maintenance of the hip flexor function would be a compensa- tion mechanism in our elderly subjects.

There have been a few studies on the comparison between the elderly population and the young population in Korea. In the studies performed on the Korean population, the kinematic and the kinetic gait patterns were similar two populations, but the gait parameters showed differences among the reports. Kwon et al. [14] reported increased cadences but decreased walking speeds and stride lengths in the sixties compared to the twenties; according to their report, the ankle and the hip range of motion increased in the sixties. However, these results were contrary to the previous studies done on the elderly in the Western world. Other studies performed on the young Korean population also showed differences in gait parameters not only with previous reports from other countries but also among those studies $[15,16]$. There are some variations in the changes of the gait characteristics depending on the fitness level or the impairment in the elderly, so further study on subjects with a diverse fitness and impairment level would be necessary for delving into the questions about the limiting factors of the elderly gait and how the elderly compensate their limiting factors. Since we had no female subjects and the range of subject's age was concentrated in their sixties, further studies on the female population and other age groups are needed.

In this study, we compared the elderly walking with the young adult walking. The elderly showed a slow walking speed. Walking speed is a significant confounding factor that can change the gait parameters. By changing the walking speed to a slow, moderate, and fast mode in the young adults, we tried to find the elderly's gait characteristics that could not be explained by the speed change. We observed that the preserved cadences, the decreased APF range and power, the preserved hip flexion range and power were specific findings that could not be explained by the slowing of the walking speed. These results should be considered when performing clinical gait research with respect to the elderly population.

\section{CONFLICT OF INTEREST}

No potential conflict of interest relevant to this article was reported. 


\section{REFERENCES}

1. Prince F, Corriveau H, Hebert R, Winter DA. Gait in the elderly. Gait Posture 1997;5:128-35.

2. Bendall MJ, Bassey EJ, Pearson MB. Factors affecting walking speed of elderly people. Age Ageing 1989;18: 327-32.

3. Hamlin CR, Kohn RR. Determination of human chronological age by study of a collagen sample. Exp Gerontol 1972;7:377-9.

4. Winter DA, Patla AE, Frank JS, Walt SE. Biomechanical walking pattern changes in the fit and healthy elderly. Phys Ther 1990;70:340-7.

5. Kerrigan DC, Todd MK, Della Croce U, Lipsitz LA, Collins JJ. Biomechanical gait alterations independent of speed in the healthy elderly: evidence for specific limiting impairments. Arch Phys Med Rehabil 1998;79: 317-22.

6. DeVita P, Hortobagyi T. Age causes a redistribution of joint torques and powers during gait. J Appl Physiol (1985) 2000;88:1804-11.

7. Riley PO, DellaCroce U, Kerrigan DC. Effect of age on lower extremity joint moment contributions to gait speed. Gait Posture 2001;14:264-70.

8. McGibbon CA. Toward a better understanding of gait changes with age and disablement: neuromuscular adaptation. Exerc Sport Sci Rev 2003;31:102-8.
9. Elble RJ, Thomas SS, Higgins C, Colliver J. Stridedependent changes in gait of older people. J Neurol 1991;238:1-5.

10. Lelas JL, Merriman GJ, Riley PO, Kerrigan DC. Predicting peak kinematic and kinetic parameters from gait speed. Gait Posture 2003;17:106-12.

11. Kim DY, Park CI, Choi YS, Moon JY, Lim JY, Kim DY, et al. Effect of slow walking speed on gait. J Korean Acad Rehabil Med 2007;31:317-23.

12. Astephen Wilson JL. Challenges in dealing with walking speed in knee osteoarthritis gait analyses. Clin Biomech (Bristol, Avon) 2012;27:210-2.

13. Judge JO, Davis RB 3rd, Ounpuu S. Step length reductions in advanced age: the role of ankle and hip kinetics. J Gerontol A Biol Sci Med Sci 1996;51:M303-12.

14. Kwon DY, Sung IY, You JY, Ha SB. 3-Dimensional gait analysis of Korean adults. J Korean Acad Rehabil Med 1998;22:1107-13.

15. Lim W, Ryu T, Choi H, Choi HS, Chung MK. A comparison of gait characteristics between Korean and Western young people. J Ergon Soc Korea 2006;25: 3341.

16. Cho SH, Park JM, Kwon OY. Gender differences in three dimensional gait analysis data from 98 healthy Korean adults. Clin Biomech (Bristol, Avon) 2004;19: 145-52. 